\title{
An acoustic image-source characterisation of surface profiles
}

\author{
P. J. Dawson*, E. De Sena ${ }^{\dagger}$ and P. A. Naylor* \\ *Electrical and Electronic Engineering Department, Imperial College, London, SW7 2AZ, UK \\ ${ }^{\dagger}$ University of Surrey, Institute of Sound Recording, Guildford, GU2 7XH, UK
}

\begin{abstract}
The image-source method models the specular reflection from a plane by means of a secondary source positioned at the source's reflected image. The method has been widely used in acoustics to model the reverberant field of rectangular rooms, but can also be used for general-shaped rooms and nonflat reflectors. This paper explores the relationship between the physical properties of a non-flat reflector and the statistical properties of the associated cloud of image-sources. It is shown here that the standard deviation of the image-sources is strongly correlated with the ratio between depth and width of the reflector's spatial features.
\end{abstract}

\section{INTRODUCTION}

The effect of rough surfaces on acoustic reflection, as well as determining the surface parameters from the projected sound field, have been points of research interest in acoustics for many years [1]. Rough surface scattering is important in the fields of sea surface [2] [3] [4] and sea bed [5] [6] mapping and in optics is a useful tool for the non destructive testing of materials [7].

A rough surface can scatter incident sound waves, provided its texture size is comparable to or much larger than the wavelength of the sound. Modelling this reflection can be done using methods such as the Boundary Element Method (BEM) [8] [9], ray tracing [10] [11] [12] or the image-source method [13] [14]. In the case of ray tracing and the image-source method, the reflections are typically assumed to be specular.

The image-source method [13] assumes that reflections on the surface are specular and models a sound field by placing additional sources in space at points where images of the source occur. The method is similar to ray tracing, but allows for a geometry of image-sources to be instantiated before knowledge of the microphones. The procedure for the method is as follows. For a given reflector and source, a second source is created at the location of the source's reflected image. The reflector itself is then removed, leaving only two sources - the true source and the image-source. For multiple reflectors the number of sources in the model is given by the number of reflectors plus the true source [15].

The model is used in room acoustics, with applications such as determining the Room Impulse Response (RIR) [16], but has also been investigated in the context of exterior scattering problems [17]. Instead of obtaining the sound field, the method discussed in this paper intends to use the image locations from the image-source method as a tool to determine parameters of a non-planar acoustically reflecting surface.
The research shown in this paper aims to explore the relationship between the distribution of image-sources invoked by a non-planar surface and the spatial parameters of the surface itself. Specifically, it is shown that the standard deviation of the image locations is an indicator of the ratio of the surface feature height and the surface feature separation for a given reflector surface profile. By learning the relationship, it can be used to derive an estimation of surface parameters from the distribution in space of the image-sources in the model.

Since the focus of this paper is on the relationship between image-sources and spatial features of the reflector, it is assumed here that a clairvoyant estimator is available for the position of the image-sources. The estimation of the positions can for instance be carried out using sparsity-inducing methods such as the one proposed by Mignot et al. [18], or, in case of convex reflectors, the method of Dokmanic et al. [19]. The estimation under real-life, noisy conditions will lead to errors in the position of the image-sources, or to some image-sources missing altogether. An analysis taking into account these losses is left for future work.

Furthermore, as in other geometric-acoustics-based methods, it is assumed that the wavelength of the impinging wave is much smaller than the size of the surface features [1] [3] [20] (i.e. $k a \gg 1$, where $k$ is the wavenumber and $a$ is the average feature size in metres). In other words, it is assumed that the specular component of the reflected field is dominant [21].

In Section II, some deterministic surface examples are given and it is shown that the spatial distribution of image-sources holds information on given spatial parameters of the surface. Then in Section III, statistical methods are introduced to present a more general relationship between the image-source distribution and a surface's spatial components. In Section IV the results of the statistical methods are discussed, as well of limitations of the findings.

\section{IMAGE POSITION ANALYSIS}

Three deterministic surface profiles are considered for image distribution analysis. The first is a castellation profile, a pattern similar to the parapets of a Norman castle with protuding periodic rectangular features. The second is a sawtooth profile, with protuding periodic triangular features. The third is a sinusoidal profile, whose height varies as a sine wave over the $x$ direction. 


\section{A. Castellation profile}

The castellation profile, shown in Figure 1, is a shape that constists of rectangular features protuding from a flat base. In this example, each rectangle has a depth $d$ and a width $w$, and are periodic with a separation distance equal to the rectangle width $w$. The vertical edges induce images at the height of the source, and are not visible to a sensor above the surface. As such, they are discarded for the purposes of position analysis.

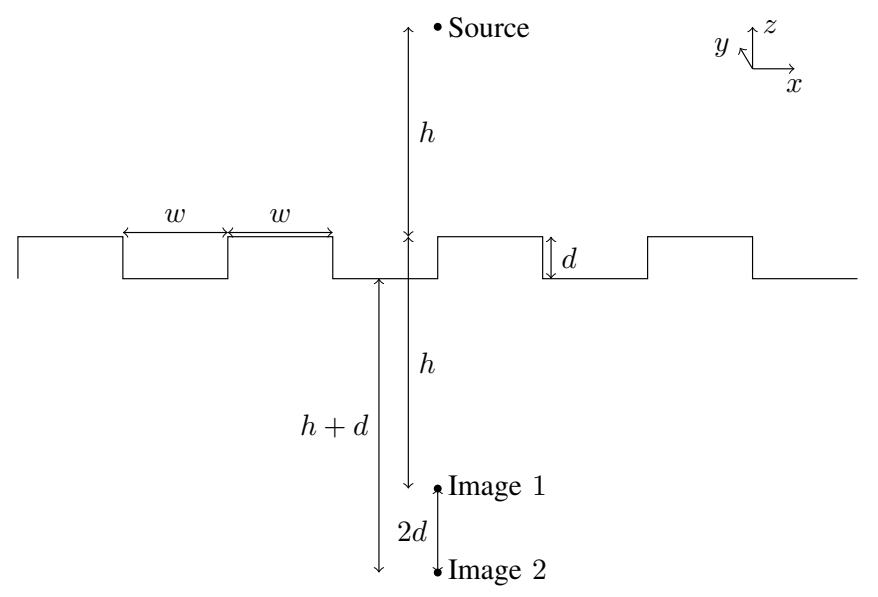

Fig. 1: Images created by the two planes of a castellation surface

The images of interest are those induced by the horizontal edges of the surface. Because they lie on two distinct planes, exactly two images are created, as shown in Figure 1. The two images are separated by a distance $2 d$. The image positions therefore hold information on the surface profile. Given knowledge of the image positions and of the profile type, it is possible to determine the height of the castellation's features. This calculation is independent of the height of the source. The horizontal parameter $w$ is not obtained from these images, but can be extracted from images created by the vertical edges.

\section{B. Sawtooth profile}

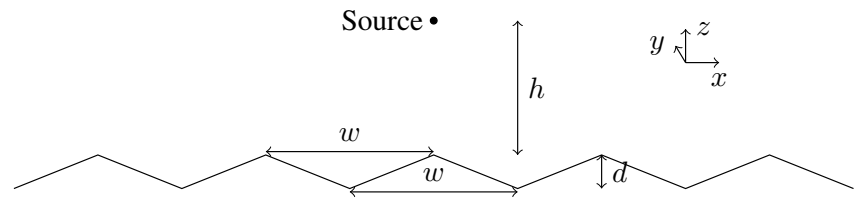

Fig. 2: Geometry of a sawtooth surface defined by its peak separation $w$ and the feature height $d$

The sawtooth profile, shown in Figure 2, consists of a series of periodic triangular features. All edge lengths are equal. Peaks are separated from each other by a distance $w$ and the peak to trough distance is $d$. The gradient at each edge is $g_{e}= \pm \frac{2 d}{w}$. The parallel edges have a perpendicular separation given in (1) and shown in Figure 3.

$$
s_{e}=\frac{w d}{\sqrt{d^{2}+\frac{w^{2}}{4}}}
$$

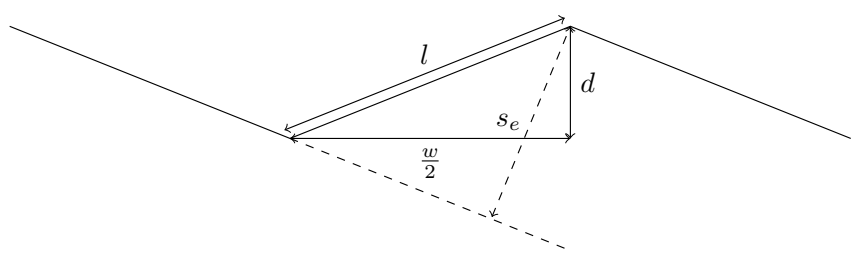

Fig. 3: Separation of sawtooth planes

Each feature on the surface consists of two edges and so will induce two separate images. If each feature is given an index $n=\ldots,-1,0,1, \ldots$ and a source is placed $h$ above the peak of feature $n=0$, then a projection matrix is formed,

$$
\begin{aligned}
\mathbf{P} & =\frac{\mathbf{a}^{T}}{\mathbf{a}^{T} \mathbf{a}} \\
& =\frac{1}{d^{2}+\frac{w^{2}}{4}}\left(\begin{array}{cc}
\frac{w^{2}}{4} & \pm \frac{d w}{2} \\
\pm \frac{d w}{2} & d^{2}
\end{array}\right),
\end{aligned}
$$

where the \pm symbol refers to projection from the left and right edges of a triangle respectively. The two images are then positioned as shown in (3) [19].

$$
\begin{aligned}
\mathbf{y} & =\left(\begin{array}{c}
n w \\
d
\end{array}\right)+(2 \mathbf{P}-\mathbf{I}) \mathbf{x} \\
& =\left(\begin{array}{c}
2 n w-\frac{\frac{n w^{3}}{2} \mp d w h}{d^{2}+\frac{w^{2}}{4}} \\
d-h+\frac{2 d^{2} h \mp n d w^{2}}{d^{2}+\frac{w^{2}}{4}}
\end{array}\right)
\end{aligned}
$$

A sawtooth surface and its image positions are shown in Figure 4. Two lines of discrete images are present, one from the positive edges and one from the negative edges. The gradient of the lines is $g_{i}=\mp \frac{w}{2 d}=-\frac{1}{g_{e}}$, and so is perpendicular to the edges that formed them. Therefore the orientation of each edge can be determined directly from the



Fig. 4: Locations of images from a sawtooth shaped reflecting surface with $d=0.08, w=1$ and the source height $h=1.5$. Note that all same coloured edges are parallel. 
gradient of the images produced. The spacing between the images on the same line is $s_{i}=\frac{2 w d}{\sqrt{d^{2}+\frac{w^{2}}{4}}}=2 s_{e}$, giving information on the perpendicular separation between each parallel edge. Together with the edge orientation, both the $w$ and $d$ parameters of the profile features can be determined.

\section{Sinusoidal profile}

The third deterministic surface is sinusoidal, and has a peak to peak displacement $d$, and a peak separation of $w$. The $z$ axis amplitude of the surface is given as

$$
z=\frac{d}{2} \cos \left(2 \pi \frac{x}{w}\right)
$$

Because the gradient of the surface is continuous, the images are a continuous line instead of discrete points. This is demonstrated in Figure 5, where three sinusoidal surfaces with differing parameter ratios are shown with their corresponding image lines from a source at height $h=1.5$.

Figure 5 shows that the image line grows from a small starlike structure in $5 \mathrm{a}$ round to a collection of near circles in $5 \mathrm{c}$ as the parameter ratio $\frac{d}{w}$ increases. Figure 5a shows a surface that more closely represents a flat surface, and Figure 5c shows a very erratic surface with features of high spatial frequency. The two are extreme cases and included for clarity. It is visible in Figure $5 \mathrm{~b}$ that the image distribution shows features similar to those in the castellation and sawtooth examples.

The line crosses two distinct points at $x=0$, induced by peaks and troughs respectively. The positions of the crossover points are as they would be in the castellation example with the same parameters, and so these points can be analysed to determine the height $d$ of the sinusoid.

The tips of the wings of the image line are produced by the points of greatest gradient on the surface. The gradient extremes are $g_{e}= \pm \frac{d \pi}{w}$. Each tip lies on one of two lines, and are periodic along these lines. This is similar to the images from the sawtooth surface, and the lines on which the wing tips lie are perpendicular to the gradient extremes, such that the gradient of the lines is $g_{i}=\mp \frac{w}{d \pi}$. The sections of the image line that spans between wing tips approximate as arcs.

\section{STATISTICAL ANALYSIS}

The surfaces discussed in Section II have been deterministic, and so the images have been trivial to calculate and observe patterns for. For a randomly generated surface, the images too are randomly distributed, and so statistical approaches are used. The focus in this section will be on the relationship between the variance and the surface parameters, specifically the ratio between the feature height $d$ and the separation $w$. This is performed for the sinusoidal surface to give a deterministic example, and a randomly generated surface. A fixed surface size is used throughout, the total width is $6 \mathrm{~m}$ for all surfaces.

\section{A. Sinusoidal surface}

The surface is instantiated as a 5000 point sinusoid in the range $x=[-3,3]$, with its $z$ values calculated using (4). The

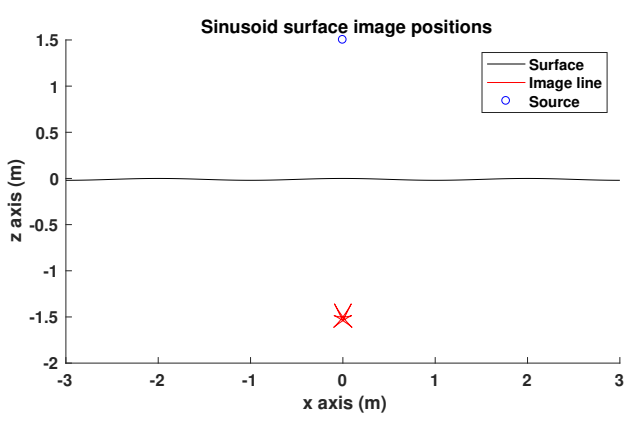

(a) $d=0.02, w=2$ and $\frac{d}{w}=0.01$

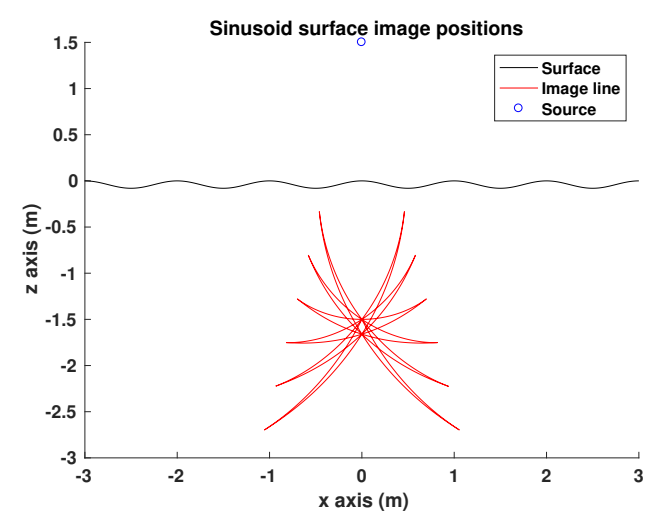

(b) $d=0.08, w=1$ and $\frac{d}{w}=0.08$

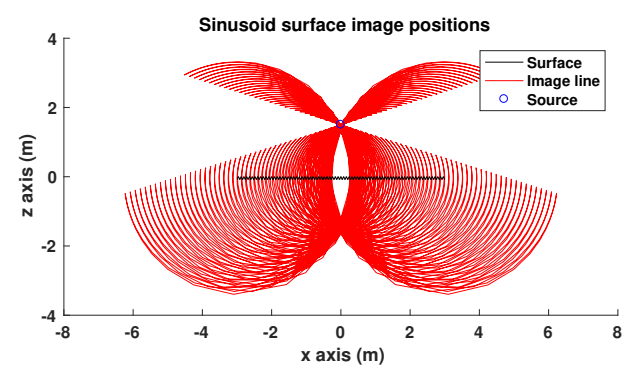

(c) $d=0.08, w=0.08$ and $\frac{d}{w}=1$

Fig. 5: Locations of images from a sinusoid shaped reflecting surface with the source height $h=1.5$ above the surface, and varying parameters $w$ and $d$ to present the effect of varying the parameter ratio $\frac{d}{w}$

parameters $d$ and $w$, described in Section II-C, are varied with 30 logarithmicly spaced values of each parameter chosen such that they lie in the range $w \in[0.0032,1], d \in[0.0032,1]$. A source is placed $1.5 \mathrm{~m}$ above the centre of the surface. Once instantiated for a given $d$ and $w$, the 4999 images are found, and the overall variance of the image positions is found. For 
each simulation, the value of $\frac{d}{w}$ is plotted against the variance $\sigma^{2}$ of the image positions, shown in Figure 6 as a log-log plot.

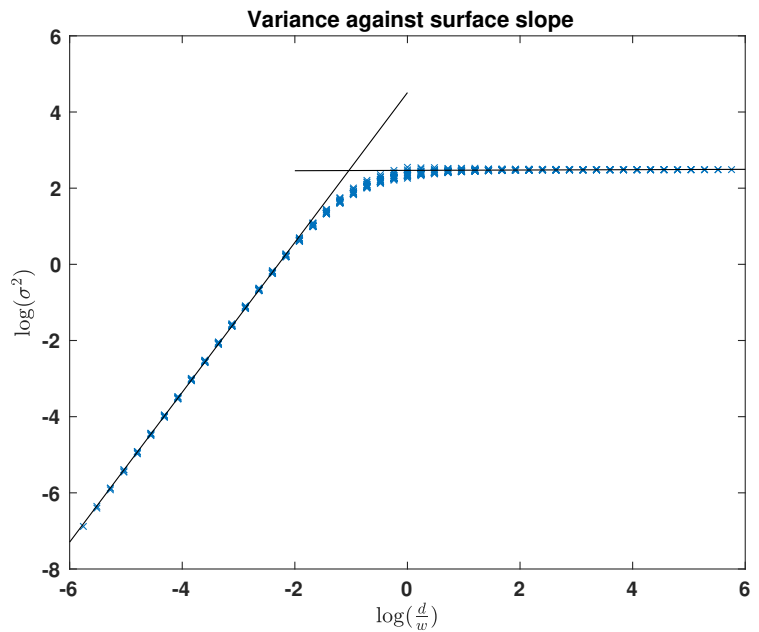

Fig. 6: Log-log plot of the variance of image positions against the parameter ratio of the sinusoidal surface, here defined as $\frac{d}{w}$, and the lines of best fit for the upper and lower section of the graph

In the range $\frac{d}{w}>1$, the variance plateaus to a single value. The line of best fit through this section is estimated to have the equation $\sigma^{2}=11.8\left(\frac{d}{w}\right)^{0.00451}$. This can be attributed to the increase of parameter ratio causing the arcs to approach the shape of circles. as shown in Figure 5c, observed to pass through the source, and overall not increasing their range from the mean image position with the parameter ratio. This type of surface is very extreme and in practice would be difficult to resolve image positions for. For this reason, the focus is on surfaces with a parameter ratio range of $\frac{d}{w} \leq 1$.

In this range, there is a positive correlation between the parameter ratio and the variance. The line of best fit for this section has an estimated equation $\sigma^{2}=90.9\left(\frac{d}{w}\right)^{1.97}$. This suggests there is a strong correlation between the parameter ratio $\frac{d}{w}$ in this range and the standard deviation $\sigma$ of the image positions.

\section{B. Random surface}

A random surface is now considered. Like all of the above surfaces, this surface is defined for a profile in the $x-z$ plane which is constant in the $y$ axis. The surface ranges from $x=[-3,3]$. Generation of the surface uses two parameters $w$ and $d$, as with the previous examples and using the same ranges. The parameter $w$ defines points along the surface at which a height value is chosen from a normal distribution with mean 0 and standard deviation $d$, the second parameter. 5000 linearly spaced sampling points are then chosen along $x$, and linear interpolation is used to find the intermediate points. An example is shown in Figure 7, where $w=0.1$ and $d=0.05$.

A source is placed $1.5 \mathrm{~m}$ above the centre of the surface, and surfaces are instantiated using the same values for $d$ and $w$ as used for instantiating the sinusoidal surface above. The image

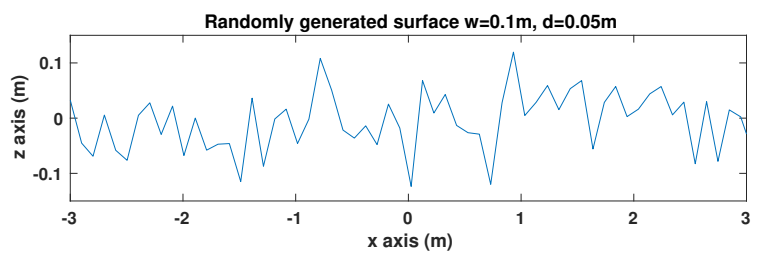

Fig. 7: Example of a randomly generated surface with parameters $w=0.1$ and $d=0.05$. The $z$ and $x$ axes have a different scale to emphasise features.

position of each of the 4999 surface edges is then calculated. Figure 8 plots the variance of the image positions against the parameter ratio $\frac{d}{w}$. Note that this is not directly related to the individual edge gradients.



Fig. 8: Log-log plot of the variance of image positions against the parameter ratio of the random surface, here defined as $\frac{d}{w}$, and the lines of best fit for the upper and lower section of the graph

The plot shows a similar trend to the deterministic example in Figure 6, in that where the parameter ratio $\frac{d}{w}<1$ there is a positive correlation between parameter ratio and the variance. The line of best fit for this region, as shown, has an estimated equation of $\sigma^{2}=42.0\left(\frac{d}{w}\right)^{1.95}$. Once again, there appears to be a linear relationship between the standard deviation $\sigma$ and $\frac{d}{w}$.

In the range $\frac{d}{w}>1$, a plateau effect is observed, showing that the variance does not increase with the parameter ratio in this region. The equation for the line of best fit of this region is estimated to be $\sigma^{2}=10.5\left(\frac{d}{w}\right)^{0.0328}$.

This example presents more error from the lines of best fit than the sinusoidal example. This may be the result of using a normal distribution which could produce anomalous edges which are very steep. This would have a greater effect on surfaces with large $w$, as there are fewer edges and therefore fewer image positions, meaning the variance will be more greatly skewed by an anomaly. 


\section{Discussion AND CONCLUSIONS}

The results shown above indicate a strong correlation between the standard deviation of the distribution of images created by a surface and the ratio between two parameters $d$ and $w$ of a 2D surface (or a 3D surface with a constant profile). This has been shown with both a deterministic and a stochastic surface. The implication of this is that if the image-sources of every edge can be found, then from statistical properties of the image-source distribution, the parameter ratio $\frac{d}{w}$ can be determined.

A larger value of $\frac{d}{w}$ is equivalent to steep spatial gradients between peaks and troughs of the surface. However, surfaces with a large enough parameter ratio, greater than unity, will eventually stop showing a linear relationship between parameter ratio and standard deviation of image positions. This is because at unity, the surface angles become around $45^{\circ}$, at which point the arcs representing the image positions reach as far as they can from the source, as shown in Figure 5c. These arcs form a part of an ellipse which intersect the source. Beyond this surface angle, images are formed which are closer to the source and don't provide a significant increase in variance.

The variance contains information about the parameter ratio, however it doesn't directly give information about $d$ or $w$ separately. Further statistical analysis of the image distribution may derive these surface parameters, which is a point for future work. There are also more parameters that define a surface's spatial parameters, such as the mean deviation, skewness and kurtosis of the surface [22].

\section{REFERENCES}

[1] J. A. Ogilvy, "Wave scattering from rough surfaces," Reports on Progress in Physics, vol. 50, no. 12, p. 1553, 1987.

[2] D. A. Miles, R. N. Hewitt, M. K. Donnelly, and T. Clarke, "Forward scattering of pulses from a rough sea surface by Fourier synthesis of parabolic equation solutions," J. Acoust. Soc. Am., vol. 114, pp. 12661280, Aug. 2003.

[3] L. Fortuin, "Survey of Literature on Reflection and Scattering of Sound Waves at the Sea Surface," J. Acoust. Soc. Am., vol. 47, pp. 1209-1228, May 1970.

[4] M. R. Mousavi, M. Karimi, and A. Jamshidi, "Probability distribution of acoustic scattering from slightly rough sea surface," Ocean Engineering, vol. 112, pp. 134-144, Jan. 2016.

[5] G. Steininger, J. Dettmer, S. E. Dosso, and C. W. Holland, "Transdimensional joint inversion of seabed scattering and reflection data," $J$. Acoust. Soc. Am., vol. 133, pp. 1347-1357, Mar. 2013.

[6] J. J. Shirron and T. E. Giddings, "A finite element model for acoustic scattering from objects near the ocean bottom," in Proceedings of OCEANS 2005 MTS/IEEE, pp. 1644-1651 Vol. 2, Sept. 2005.

[7] A. Fercher, U. Vry, and W. Werner, "Two-wavelength speckle interferometry on rough surfaces using a mode hopping diode laser," Optics and Lasers in Engineering, vol. 11, no. 4, pp. 271-279, 1989.

[8] L. Fu, K. Frenner, and W. Osten, "Rigorous speckle simulation using surface integral equations and higher order boundary element method," Optics letters, vol. 39, no. 14, p. 4104, 2014.

[9] S. Chandler-Wilde and S. Langdon, "Boundary element methods for acoustics," lecture notes, University of Reading, July 2007.

[10] M. Brigante, "On multiple scattering in acoustic media: A deterministic Ray Tracing method for random structures," Ultrasonics, vol. 53, pp. 652-657, Mar. 2013.

[11] X. Wen, C. Wang, and H. Zhang, "The Scattering Study of Rough Surface by Ray Tracing Technique," in 2009 International Conference on Environmental Science and Information Application Technology, vol. 2, pp. 704-707, July 2009.
[12] H. R. Anderson, "A second generation 3-D ray-tracing model using rough surface scattering," in Proceedings of Vehicular Technology Conference - VTC, vol. 1, pp. 46-50 vol.1, 28 Apr-1 May 1996.

[13] J. B. Allen and D. A. Berkley, "Image method for efficiently simulating small-room acoustics," J. Acoust. Soc. Am., vol. 65, pp. 943-950, Apr. 1979.

[14] L. Savioja and U. P. Svensson, "Overview of geometrical room acoustic modeling techniques,” J. Acoust. Soc. Am., vol. 138, pp. 708-730, Aug. 2015.

[15] P. A. Naylor and N. D. Gaubitch, eds., Speech Dereverberation. Springer-Verlag, 2010

[16] S. Mischie, "On acoustic source localization in a reverberant environment based on image method," in Proc. Intl. Conf. on Optimization of Electrical and Electronic Equipment (OPTIM), (Bran, Romania), pp. 886-891, May 2014.

[17] S. S. Rienstra and A. A. Hirschberg, "An introduction to acoustics," lecture notes, Technische Universiteit Eindhoven (TU/e), 2004.

[18] R. Mignot, L. Daudet, and F. Ollivier, "Room Reverberation Reconstruction: Interpolation of the Early Part Using Compressed Sensing,' IEEE Trans. Audio, Speech, Lang. Process., vol. 21, pp. 2301-2312, Nov. 2013.

[19] I. Dokmanić, R. Parhizkar, A. Walther, Y. M. Lu, and M. Vetterli, "Acoustic echoes reveal room shape," Proc. National Academy of Sciences, 2013.

[20] Y.-K. Yoon and J. Kim, "KA and Ray tracing approximation on the surface roughness," Antennas and Propagation (APSURSI), 2011 IEEE International Symposium on, pp. 3355-3357, 2011.

[21] H. Kuttruff, Room Acoustics. London: Spon Press, 5th ed., 2009.

[22] "Geometrical Product Specifications (GPS) - Surface texture: Profile method - Terms, definitions and surface texture parameters," Standard ISO 4287:1997, Intl. Org. for Standardization (ISO), 1, ch. de la VoieCreuse, CP 56, CH-1211, Geneva 20, Switzerland, Apr. 1997. 